Vol. 12, No. 42, January, 2017, 91-102

\title{
PRACTICAL DESIGN OF THE FEEDBACK ELEMENT OF THE HORIZONTAL PART OF A DUAL-AXIS SUN TRACKER
}

\author{
Mahmoud S Gaballa, Mohiy Bahgat, Abdel-Ghany M Abdel-Ghany \\ Electrical Power and Machines Department, Helwan University,
}

\begin{abstract}
This paper presents a detailed design of all mechanical and electronic parts of a Deviation Measuring Unit (DMU). This unit has been used as a feedback element for the horizontal part of a dual-axis sun tracker. The proposed dual-axis sun tracker is designed to be fixed over moving surfaces such as vehicles, trains or ships. The modeling, simulation and implementation of the proposed sun tracker was earlier introduced in a previous work. Due to the fast and discontinuous change of the relative sun direction in the horizontal plane, a need arose for having a feedback element with special specifications among the control process. The designed DMU proven to meet all the desired specifications and concerns to satisfy the special needs for the designed tracking control system.
\end{abstract}

Keywords: Sun Tracking System, Deviation Measuring Unit, DMU, Fast-Response Sun Tracker, Discontinuous Tracking, Dual-Axis Sun Tracker, Moving Sun Tracker

\section{INTRODUCTION}

Deploying a sun tracker over moving surfaces is rather a challenge due to the arising of new different circumstances related with the motion, such as the unexpected change in geo-location, the sudden change in sun direction and the high possibility for the sun tracker of being repeatedly shaded during day hours. In a previous work [1] an experimental model for the A dual-axis sun tracker over a movable structure was presented, see Fig (1).

The error signal in the horizontal-motion control process is the angular deviation of the sun beam from the perpendicular direction. The Deviation Measuring Unit DMU is a module that is responsible of acquiring the error of sun position and passing it as a feedback signal to the controller as displayed in Fig (2). In this work, the main concerns about the DMU are:
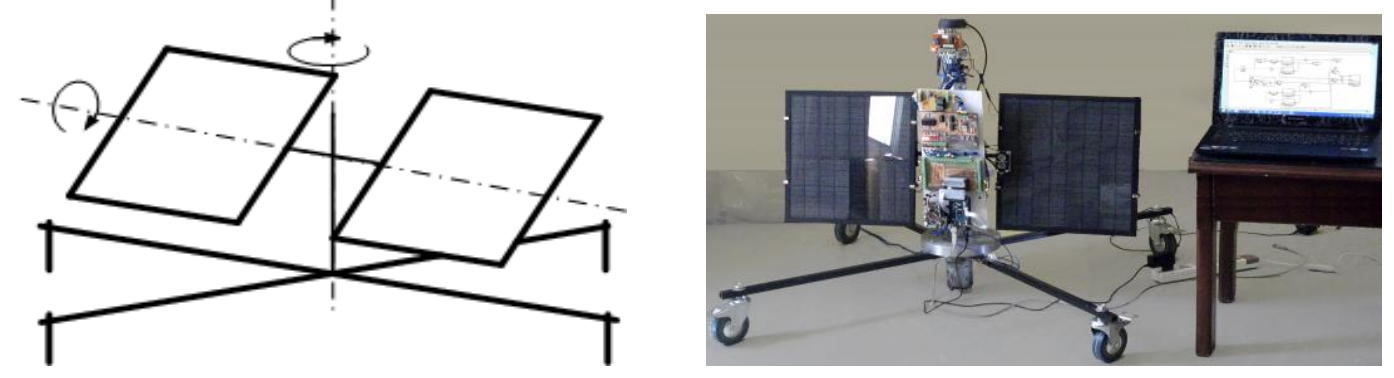

Fig (1) - A Dual-Axis Sun Tracker Over A Movable Structure 
1- To have as small as possible processing time so that the resulted delay that affects the control loop can be neglected.

2- Not to depend on having the change in sun position be gradual or continuous, as the sun tracker is desired to be used in moving entities that can suddenly change their directions, and the controller is needed to keep track of the sun position whatever the speed of its change.

3- To maintain the last detected angular position of the sun in cases of being completely shaded, for example, when passing under a bridge or beside a high building.

4- Its output should linearly represent the deviation angle.

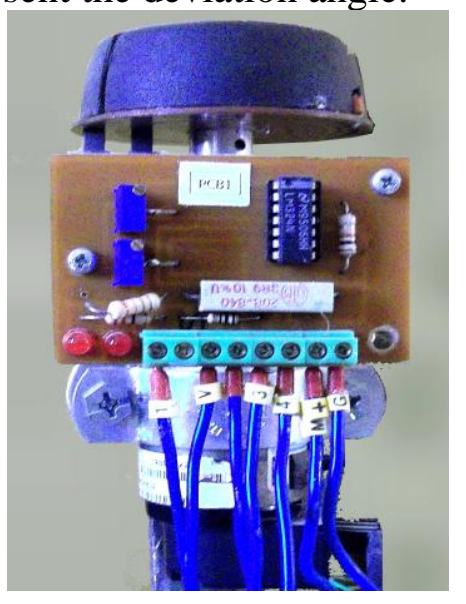

Fig (2) - The Deviation Measuring Unit DMU

Many other studies handled some of those issues in different ways, but none of them satisfied all the above concerns. In [2,3] the technique of position detecting depends on performing an exploring motion using the PV panels itself, as a photo sensor, towards any arbitrary direction the controller then compares the PV output power after and before the exploring motion. If the power is increasing then it continues moving the panels in the same direction, otherwise it moves in the opposite direction. It's found that the studies in [2,3] don't satisfy most of the above concerns as follows as it consumes a long processing time causing a long delay in the control process, it also strongly depends on having the sun position changing gradually, i.e. the controller may suddenly loses tracking if the sun position changed rapidly by an angle larger than $90^{\circ}$. It doesn't maintain the last detected sun direction.

Studies in [4-7] depend on the "Auxiliary Bifacial Solar Cells" technique, an opaque barrier separates two photo-sensors, and the outputs $\mathbf{S}$ from the two sensors $\mathbf{s}_{\mathbf{1}}$ and $\mathbf{s}_{\mathbf{2}}$ is calculated $\mathbf{s}=\frac{\mathbf{s}_{1}}{\mathbf{s}_{2}}$, this is illustrated in Fig (3). The techniques in[4-7] are very simple, very fast response and has approximately no power consumption, but still has two major problems which are: it easily losses tracking if the sun position becomes $>90^{\circ}$, i.e. somewhere behind the barrier, where boths $\mathbf{s}_{\mathbf{1}}$ and $\mathbf{s}_{\mathbf{2}}$ become shaded acquiring the same signal values and it doesn't maintain the last detected sun direction.

Fig (3) - Auxiliary Bifacial Solar Cells

The study in [8] shows a different technique that depends on having a predefined database of sun locations during the year. The study in [9] utilized the same technique as in [8] in addition to a closed loop controller to compensate for the error that occur due to time drifts (and/or) 
faulty installation. The technique in [8,9] doesn't fit in case of moving entities because it strongly depends on having the panels located in a fixed and predefined geo-location along the year, it also has a disadvantage that it needs a relatively large memory size to maintain perminute sun positions along the year.

The studies in [10,11] utilized the so called Micro-Electro-Mechanical Systems technologies (MEMS) where the proposed sensor includes two photo-diodes, a metal shield with a hole partially cover the sensors as shown in Fig (4).
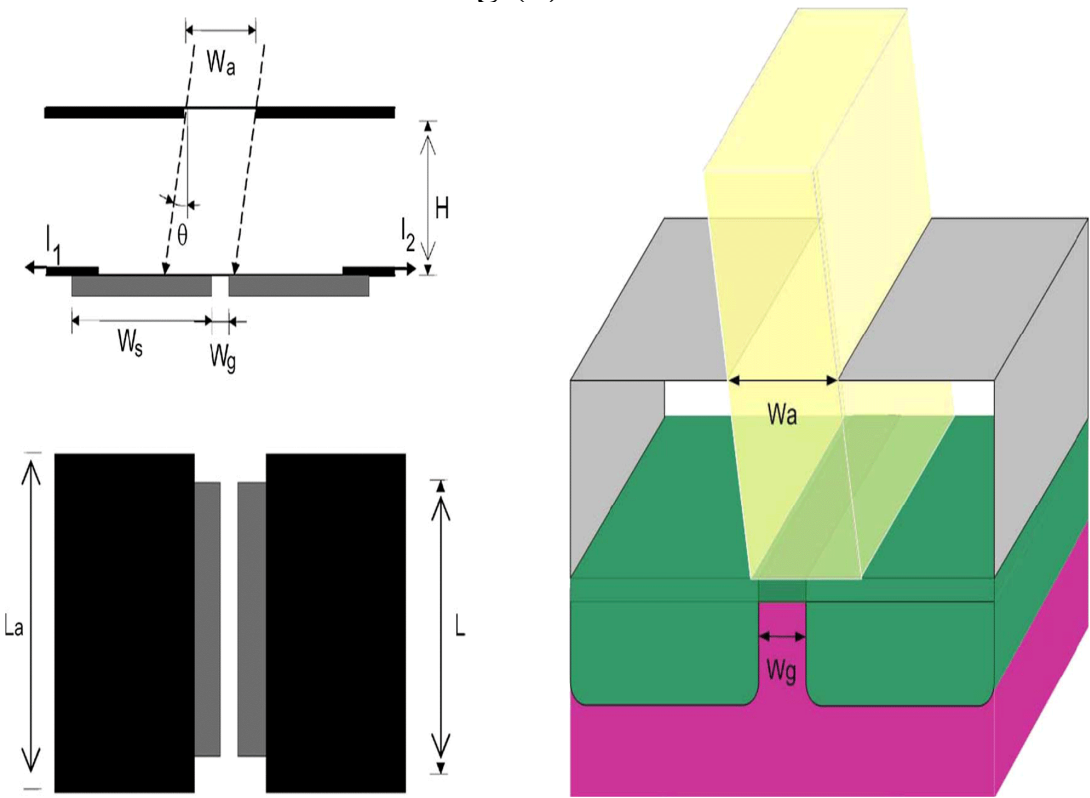

Fig (4) -Micro Electro-Mechanical System (MEMS) Deviation Sensor

A percentage of the area of each photodiode is exposed to the light falling on the sensor. Each photodiode generates electric current that is proportional to its illuminated area. The illuminated areas are determined by the shadows dropped over the photodiodes which depend on the angle of incident the light beam. This technique has the same advantages and disadvantages of the technique used in [4-7] regarding the four special concerns mentioned above, but it still has one more advantage that is the photo sensors are compact within one small package.

The studies in [12-16] utilized two separate potential dividers each consist of an LDR photosensor and a fixed resistance instead of utilizing one potential divider as in [4-7]. This technique also doesn't satisfy all of the four above mentioned concerns as it is very much like the technique used in [4-7] but with one more advantage that is its output is linearly proportional with the value of the sun direction as the two separate output voltages are passed to the microcontroller that makes the necessary calculations to generate the desired linear signal.

\section{2. .DMU Idea of Work}

The DMU technique depends on having a rotating photo-sensor that continuously scans the $360^{\circ}$ space surrounding the unit seeking for the highest light intensity beam, meanwhile, the rotation angle is being measured by a digital shaft encoder so that when the rotating sensor detects the targeted light beam the measured rotation angle corresponding to the sun position is then passed to the controller as a binary feedback signal. The DMU design satisfies the above-mentioned concerns. 


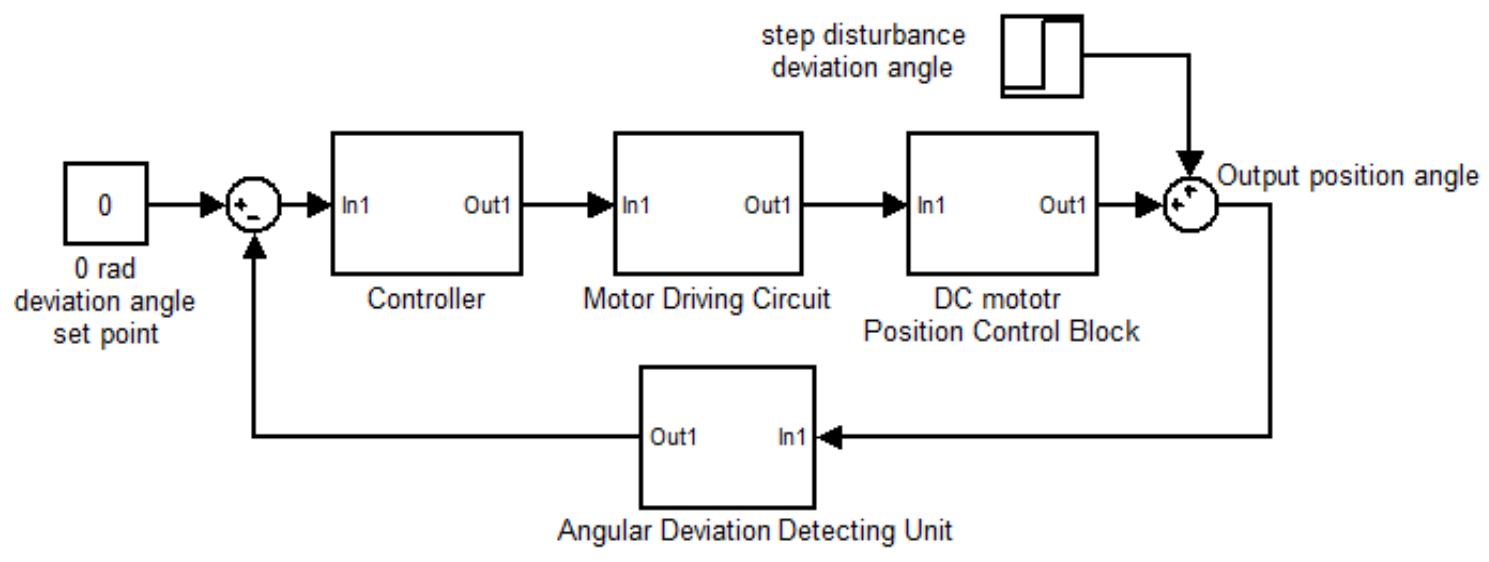

Fig (5) - Sun Tracker Control Diagram

The first concern to be mentioned here is to have the delay in the control loop as small as possible. Among all studied deviation detectors, the proposed designed DMU has a special source for delay which is the time taken by the rotating element to reach the position of the sun direction. The value of the delay time is a random value in the closed period [0 sec, $1 / n \mathrm{sec}$ ] where $\boldsymbol{n}$ is the number of revolutions per second. Actually, the rotation angular velocity of the DMU is relatively high - (about $8 \mathrm{rev} / \mathrm{sec}$ ) - which means that any change in sun position can be detected within a maximum of 125 milliseconds, and accordingly having a small processing time which means that the delay that affects the control loop can be neglected. The delay effect on the time response of the control process was studied using four values for the rotation speed which are: 8 revolutions/sec, 6 revolutions/sec, 4 revolutions/sec and 2 revolutions/sec as illustrated in Fig (6).

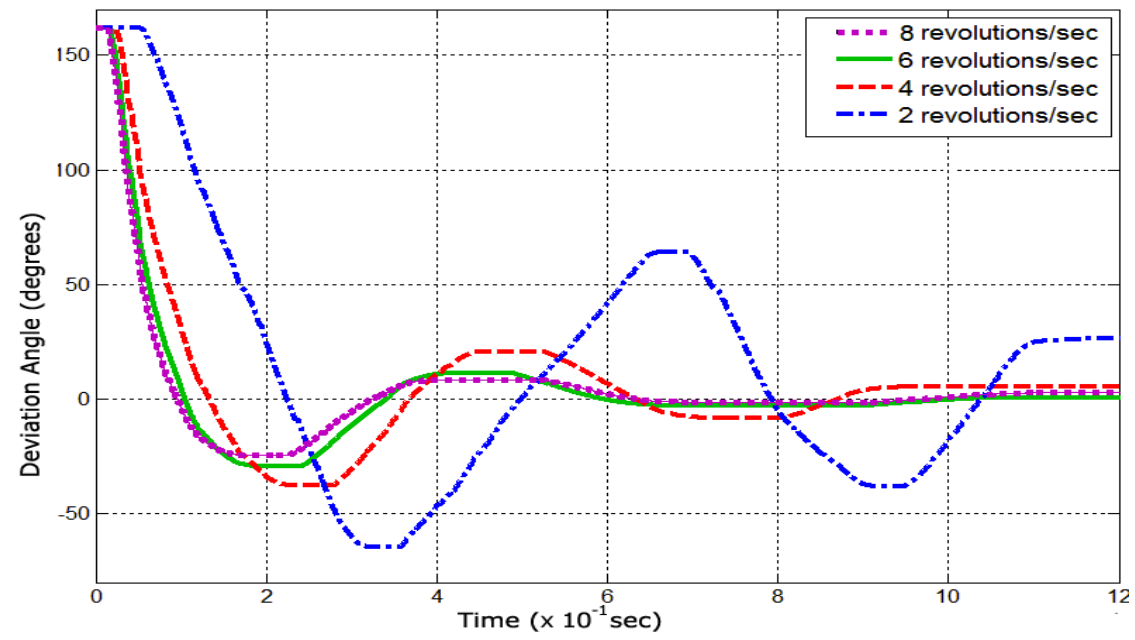

Fig (6) - Delay Effect on process response

The second concern to be mentioned is how the DMU handles the behavior of discontinuous position change of the sun. Due to the $360^{\circ}$ rotation of the photo-sensor and the relatively high rotation speed, the DMU is always able to determine the position of the sun wherever it is and whatever the speed the position is changing, i.e. it doesn't depend on having gradually changing in the sun position.

The third concern is the maintainability of the last acquired sun direction in cases of temporary losing sun light if being shaded while passing under bridges or beside high buildings. The purpose of this concern is to prevent the system from randomly moving seeking for the sun dissipating power for nothing while being shaded, the system instead, has to stop moving 
keeping its PV panels directed towards the last detected direction before the shading. The DMU as will uses a binary buffer to keep the binary value corresponding to the detected sun position and it never change this value unless it receives another value for the sun position, i.e. it maintains the position of the PV panels as it is during the period of darkness or shading and resumes changing the direction values as soon as it's being exposed to sun light again (this will be explained in section III-C). This technique also has another advantage in consuming as minimum power as possible where the last detected position is most probably be the same as the new one or at least near to it resulting in small panel correcting movement and accordingly, the power consumption will be minimum.

The fourth concern is a very essential point in control processes where it is desired to have the feedback signals linearly represents the actual values, otherwise, the function of the proportional controllers will show very unexpected behaviors causing undesired responses an $\boldsymbol{R}_{2}$ even

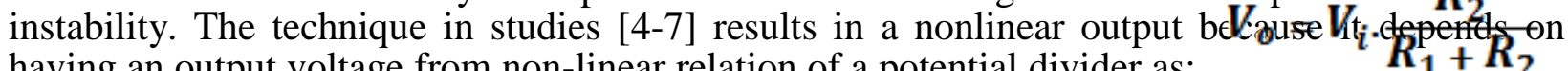
having an output voltage from non-linear relation of a potential divider as:

where $\boldsymbol{V}_{\boldsymbol{o}}$ is the output voltage, $\boldsymbol{V}_{\boldsymbol{i}}$ is the input voltage and $\boldsymbol{R}_{\boldsymbol{I}}$ and $\boldsymbol{R}_{\boldsymbol{2}}$ are the two potential divider resistances. in addition, the relation between the light intensity value and the resistance value of the LDR photo-sensor is a logarithmic relation.

In this work, the output of the proposed designed DMU is linear as it represents the $360^{\circ}$ of the horizontal deviation angle by a 100 binary values $[0,100]$, i.e. output $^{\text {out }}=\frac{\mathbf{1 0 0}}{\mathbf{3 6 0}} \cdot \boldsymbol{\theta}$, where $\Theta$ is the deviation angle.

\section{3.. DMU Design Description \\ A. Rotating Element}

The rotating element is a small opaque cylinder with a longitudinal cavity such that when it is only being directed to the sun, it permits a narrow light beam to pass to a small photo resistance $\mathbf{R}_{\mathbf{p}}$ located ingide the cylinder on its axis line, see Fig.(7). A potential divider consisting of the photo resstance $\mathbf{R} \mathbf{p}$ and another fixed-value resistance Ro is used to generate a variable voltage $\mathbf{V}_{\mathbf{p}}=\left(\mathbf{R}_{\mathbf{p}}+\mathbf{R}_{\mathbf{o}}\right)$ that increases with the direct light beam incidence as $\mathbf{R}_{\mathbf{p}}$ is inversely proportional to light intensity.

For the purpose of fine-tuning the sensor so that it only responds to sun light and neglects the light sources of smaller intensities, the output voltage $\mathrm{V}$ is then passed to an operational amplifier Op-Amp analogue comparator (LM324) as a non-inverting input while the inverting input is connected to output voltage of the tuning potentiometer $\mathrm{P}_{1}$ with the value of $\mathbf{V}_{\mathbf{p}}$.

For the purpose of wiring the photo resistance while rotating, two copper slip-rings with two carbon brushes are used.

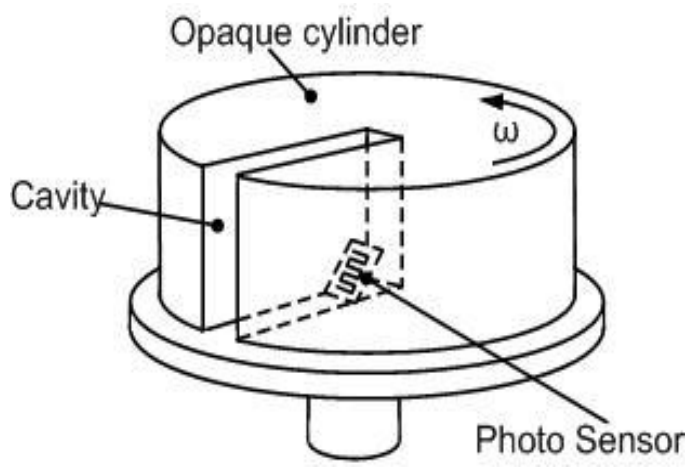


Fig (7) - The DMU rotating element

\section{Position Encoder}

This unit is used for generating square pulses during the rotation of the rotating element where the count of the pulses reflects its angular position. The encoder used consists of two punched rotary disks, each rotates across a photo-interrupter.

The first disk contains 100 punches along the disk circumference and is used to generate a pulse train of 100 square pulses per revolution to be used to trigger a counting circuit that precisely determine the angular position of the rotation as displayed in Fig (8).

The other disk contains one punch along its conference, and is used to generate an index signal (0 signal) per revolution to reset the counter after each revolution. The detailed design drawing are set in Appendix 1.
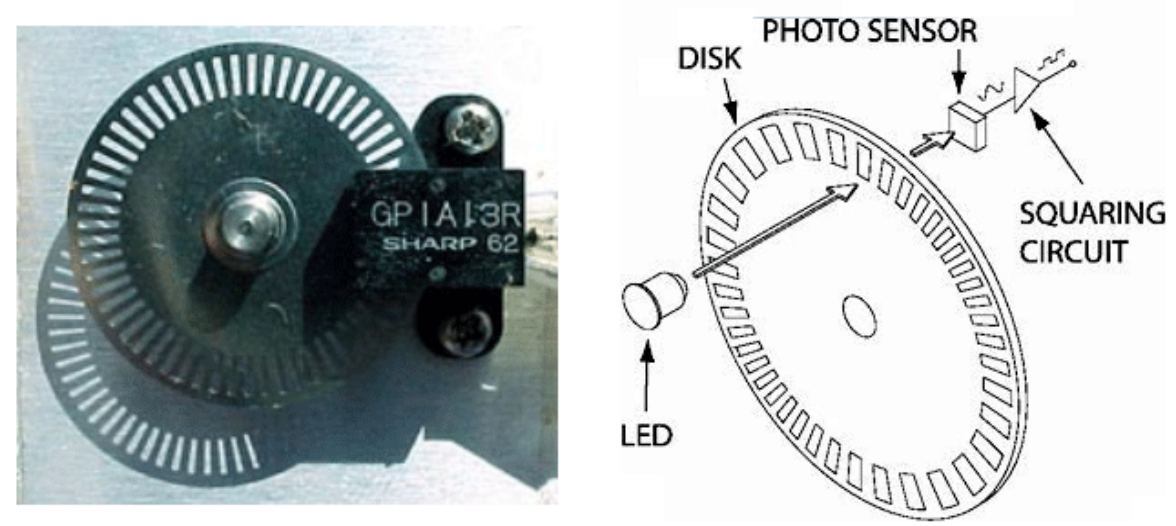

Fig (8) - Rotary-Disk Encoder

\section{B. Position Determining Circuit}

The position determining circuit shown in Fig (9) is the responsible circuit for precisely generating a binary number from 0 to 100 corresponding to the light deviation angle which is also called error value from the zero position (set point). The zero position is obtained when the light is perfectly perpendicular to the PV panels. Such a circuit consists of a dual 4-bit binary counter IC 74393 that counts the pulses generated by the position encoder disk and is reset every one complete revolution by means of the index signal generated by the index disk.

The counter IC continues counting from 0 to $100(0000000 \mathrm{~b}$ to $1100100 \mathrm{~b})$ then restarts again from 0 and so on. The 7-bit binary output of the counter is passed to the inputs of a binary latch IC 74116.

The 74116 is a dual 4-bit latch with each latch having an independent asynchronous clear input and a gated two-input enable circuit. When both enable inputs are low, the output levels will follow the data input levels. When either one or both of the enable inputs are taken high, the outputs remain at the last levels step at the inputs prior to the low-to-high transition at the enable input(s). After this, the data inputs are locked out. The detailed waveform is displayed in Appendix 2.

The clear input is overriding and when taken low will reset all the four outputs to low regardless of the levels of the enable inputs. In this study the 74116 latch is used such that it holds the input values at its outputs whenever it receives an enabling signal generated from the rotating photo sensor, the latch output remains unchanged with the change of its input values. The binary value held on the latch output is corresponding to the count represents the angular position of the light beam. 

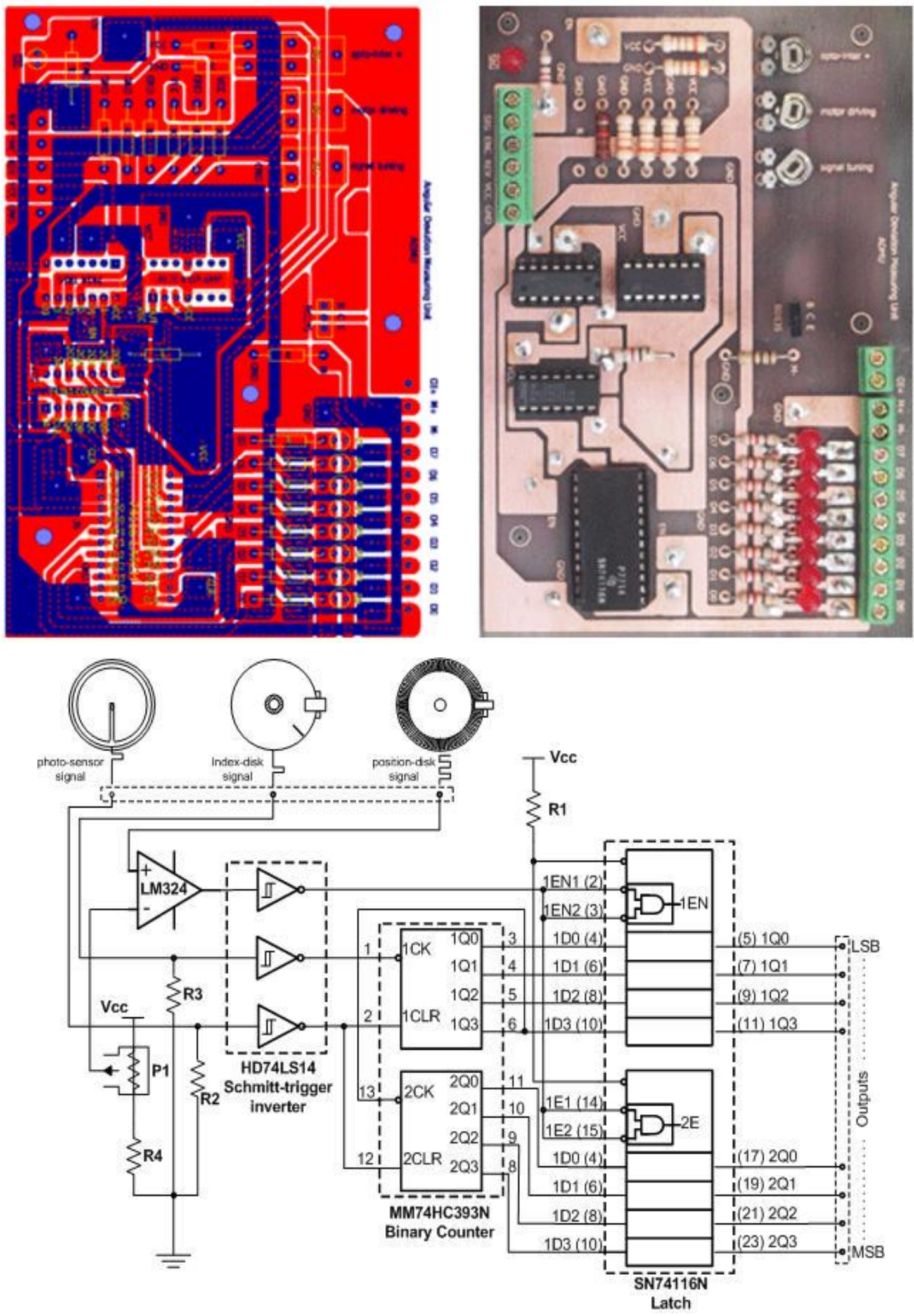

Fig (9) -Position determining circuit

\section{CONCLUSTION}


It is an important need to have a feedback element with special specifications in case of designing a sun tracking system constructed over moving entities such as vehicles or etc. In this work, many other solutions were studied and it was found that none of them can fit the special needs of the case, and finally an electro-mechanical Deviation Measuring Unit DMU was designed and implemented to satisfy all the special needs of the case process which are:

1. Small processing delay.

2. Handling the discontinuous error change.

3. Maintaining the last detected sun direction in cases of being shaded.

4. Linearly representing the direction angle value.

Finally, the results of operation came very satisfying. A comparison between the surveyed tracking techniques against the four special concerns mentioned above is shown in Tab.(1).

\begin{tabular}{|c|c|c|c|c|}
\hline & 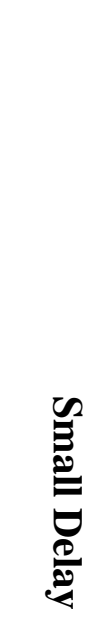 & 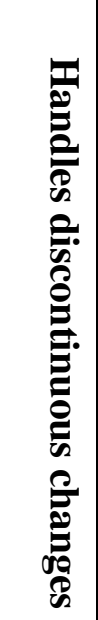 & 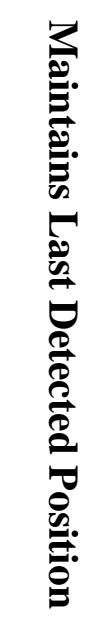 & (2) \\
\hline Exploring-Motion-Based & No & No & No & No \\
\hline Auxiliary-Bifacial Solar Cells & Yes & No & No & No \\
\hline Position-Database & - & No & No & - \\
\hline MEMS & Yes & No & No & No \\
\hline Using Two Separate Sensors & Yes & No & No & Yes \\
\hline DMU & Yes & Yes & Yes & Yes \\
\hline
\end{tabular}

Tab.(1): Comparison between the surveyed tracking techniques against four special concerns

\section{REFERENCES}

[1] Mahmoud S. Gaballa, Mohiy Bahgat, Abdel-Ghany M. Abdel-Ghany, "Modeling \& TS Fuzzy Control of a Dual-axis Sun Tracker Photo-Voltaic Panel Fixed on a Moving Vehicle", Eighteenth International Middle East Power Systems Conference (MEPCON), 2016.

[2] Daniel A. Pritchard, "Sun Tracking by Peak Power Positioning for Photovoltaic Concentrator Arrays", Sandia National Laboratories, 1983.

[3]M. G. Figueiredo1, José M. G. Sá da Costa, "Intelligent Sun Tracking System for Efficiency Maximization of PV Energy Production", RE\&PQJ, Vol. 1, No.6, March 2008.

[4] V.Poulek, M.Libra, "A very simple solar tracker for space and terrestrial applications", ELSEVIER, Solar Energy Materials \& Solar Cells, 2000. 
[5] Mahmoud S. Gaballa, Ahmed Bahgat, Ahmed Saqr, "Design and Implementation of a Computer Control System for Tracking Highest Intensity Light Source", Cairo University, 2011.

[6]Jing-Min Wang and Chia-Liang Lu, " Design and Implementation of a Sun Tracker with a Dual-Axis Single Motor for an Optical Sensor-Based Photovoltaic System", Sensors 2013.

[7] Okpeki U.K., Otuagoma.S.O, "Design and Construction of a Bi-Directional Solar Tracking System", International Journal Of Engineering And Science Issn: 2278-4721, Vol. 2, Issue 5 (February 2013).

[8] John T. Agee and Adisa A. Jimoh, "Sensorless Control of a Polar-Axis PV Tracking System", InTech,October 2010.

[9] I. Luque-Heredia, F. Gordillo, F. Rodríguez, "A PI-based hybrid sun tracking algorithm for photovoltaic concentration", European Commission 2003.

[10] José M. Quero, Carmen Aracil, Leopoldo G. Franquelo, Jordi Ricart, Pablo R. Ortega, Manuel Domínguez, Luis M. Castañer, and Rafael Osuna, "Tracking Control System Using an Incident Radiation Angle Microsensor", IEEE Transactions on industrial electronics, vol. 54, no. 2, (April 2007).

[11] J. M. Quero, P. Castro, J. M. Moreno, M. Reina, P. Ortega, "SENSOSOL: MultiFOV 4Quadrant high precision sun sensor for satellite attitude control", Proc. of the Intl. Conf. on Advances In Computing, Electronics and Electrical Technology - CEET 2014.

[12] A.Sarhan, Amged El-Wakeel and A.B. Kotb, "Analysis and Control of Hybrid Stepper Motor for Automatic Sun-Tracking System", 13th International Conference on Aerospace science \& aviation technology, (May $26-28,2009$ ).

[13] Asmarashid Ponniran, Ammar Hashim, Handy Ali Munir, "A Design of Single Axis Sun Tracking System", The $5^{\text {th }}$ International Power Engineering and Optimization Conference (PEOCO2011), Shah Alam, Selangor, Malaysia: 6-7 June 2011.

[14] K. Sreenivasa Rao, M. Mahesh, "ARM Based Solar Tracking System", International Journal of Modern Engineering Research (IJMER) Vol.2, Issue.4, July-Aug. 2012.

[15] Kais I. Abdul-Lateef, " A Low-Cost Single-Axis Sun Tracker System Using PIC Microcontroller", Diyala Journal of Engineering Sciences, Vol. 05, No. 01, pp.65-78, June 2012

[16] Tiberiu Tudorache1,Liviu Kreindler, "Design of a Solar Tracker System for PV Power Plants", Acta Polytechnica Hungarica Vol. 7, No. 1, (2010). 


\section{APENDECES}

\section{Appendix1: Mechanical drawing of the DMU}

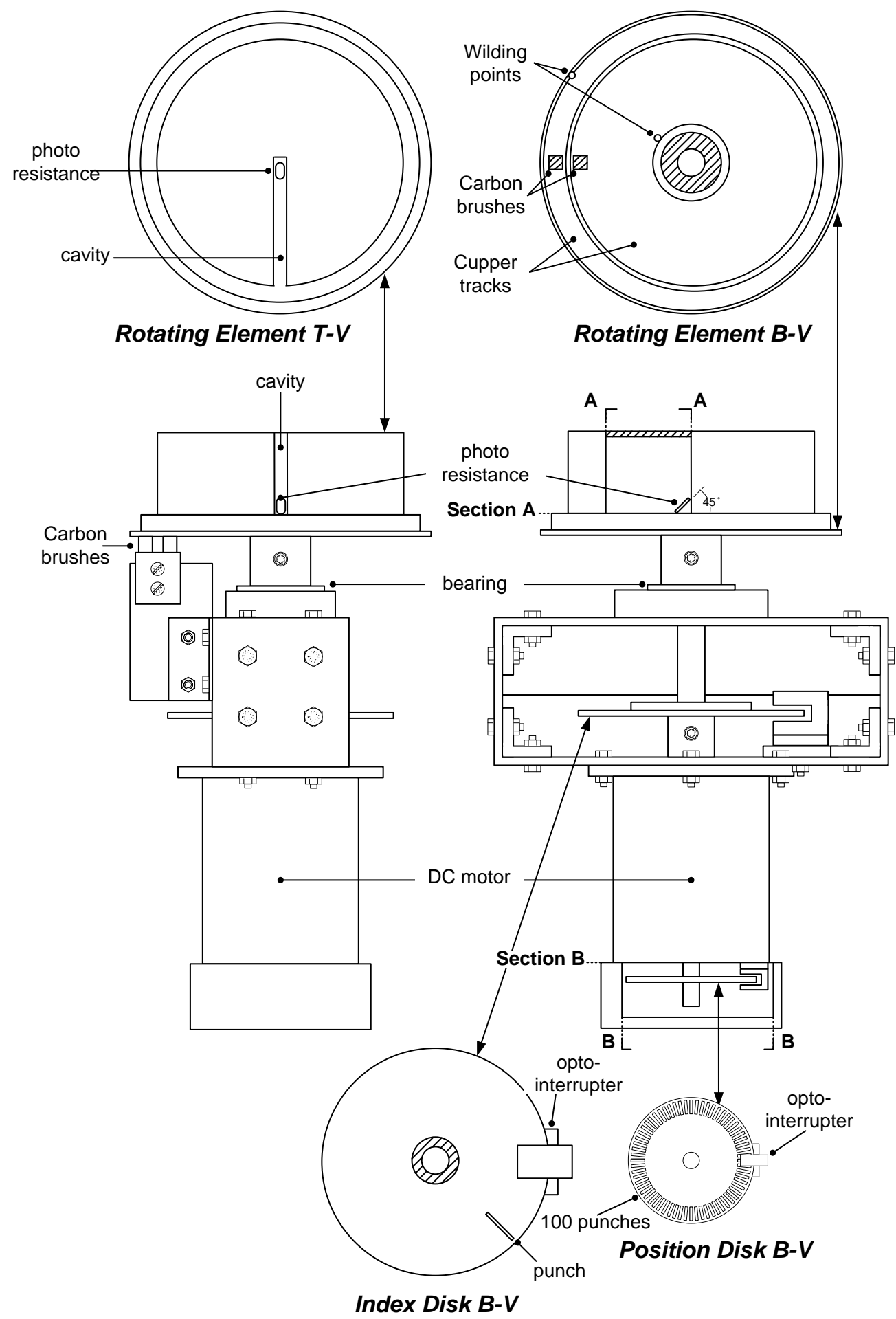

Appendix2: Signal wave form of the DMU 


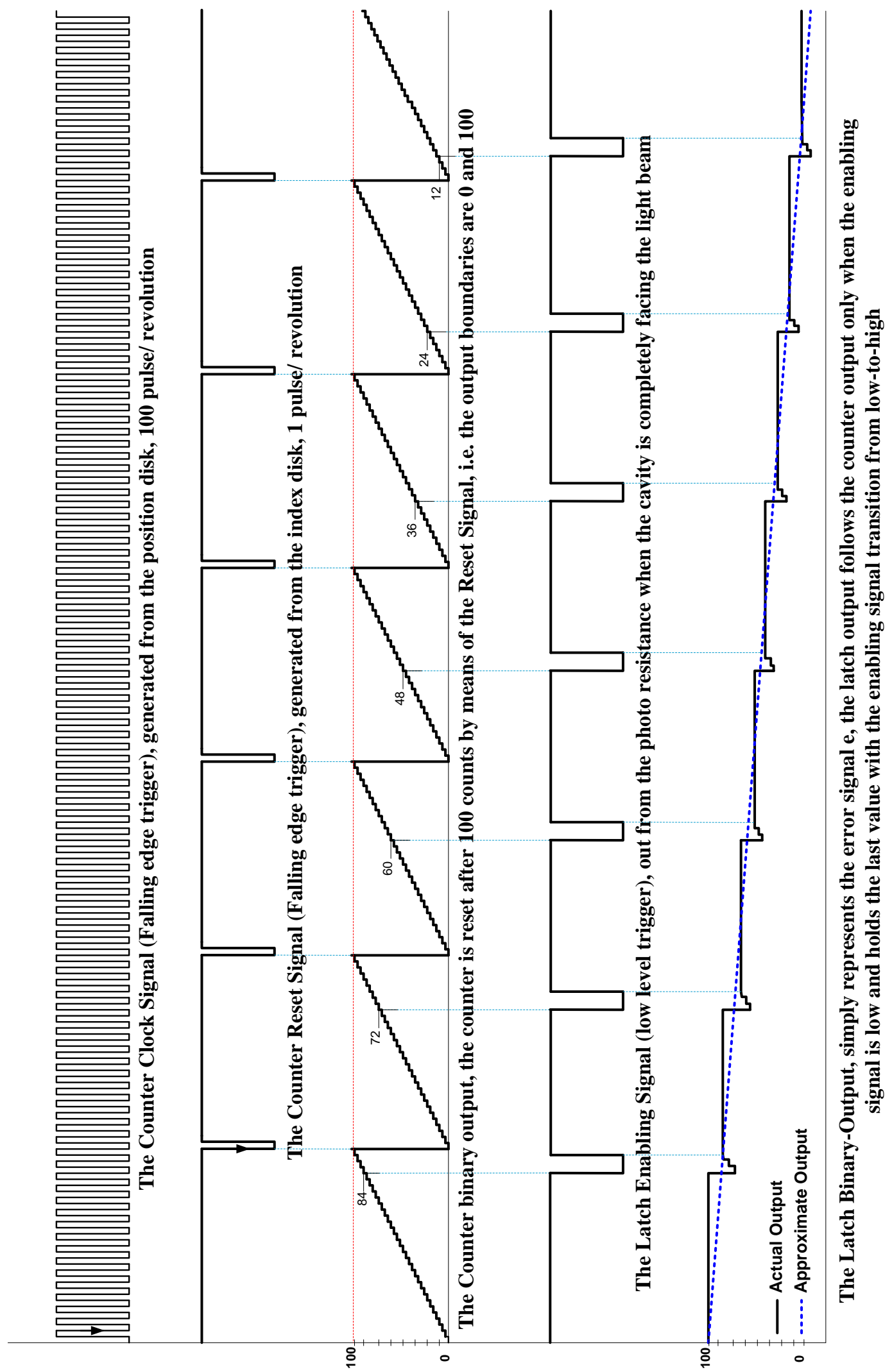

